\title{
LONG-TERM FOLLOW-UP AFTER NEONATAL COLONIZATION WITH EXTENDED SPECTRUM BETA-LACTAMASE GRAM-NEGATIVE BACTERIA
}

\author{
K. Kock ${ }^{1}$, B. Jonsson ${ }^{2}$, O. Aspevall ${ }^{3}$, A. Iversen ${ }^{4}$, C.G. Giske ${ }^{4}$, V. Nordberg ${ }^{5}$, M. Norman ${ }^{5}$, L. Navér ${ }^{5}$ \\ ${ }^{I}$ Department of Neonatology, Karolinska University Hospital, ${ }^{2}$ Department of Woman and Child Health, \\ ${ }^{3}$ Department of Laboratory Medicine, Division of Clinical Microbiology, ${ }^{4}$ Department of Microbiology, \\ Tumor and Cell Biology (MTC), ${ }^{5}$ Department of Clinical Science, Intervention and Technology \\ (CLINTEC)/Division of Paediatrics, Karolinska Institutet, Stockholm, Sweden
}

Objective: Neonatal colonization of the gut with multiresistant $(E S B L=$ Extended spectrum beta-lactamase) gram-negative bacteria has become more common in recent years. The aim of this study was to investigate whether neonatal ESBL colonization becomes long-lasting or disappears.

Methods: Follow-up study of a cohort (13 infants, 7 boys, 10 preterm) born 2008-2009 in Stockholm and colonized during neonatal intensive care with the same clone of Klebsiella pneumoniae-ESBL, as determined by PFGE. Stool cultures were performed every second month after hospital discharge until 2 years of age.

Results: Co-colonization of the gut with E.coli ESBL was seen in 9 children. Four children remained ESBLpositive at two years of age. One child was ESBL-positive at final follow-up preceded by 3 subsequent negative cultures. Two children had a negative culture at two years of age but had been ESBL-positive in one or more of the preceding 3 cultures. Six children had 3 or more subsequent negative cultures and remained culture negative at the last sampling occasion. One patient with severe lung disease died. During the 2-year follow-up, one infant was treated for a lower urinary tract infection caused by K.pneumoniae-ESBL. No other infection caused by ESBL-producing bacteria occurred.

Conclusion: Neonatal colonization with ESBL-producing gram-negative bacteria may be a transient condition for some infants, but results in long-lasting colonization or re-colonization in about $50 \%$. No infant suffered from serious infection caused by ESBL-producing bacteria during the follow-up. 